\title{
Association between smoking and cancers among women: results from the FRiCaM multisite cohort study
}

\section{Angelo Giosuè Mezzoiuso}

Universita Vita-Salute San Raffaele Facolta di Medicina e Chirurgia

Anna Odone

Universita Vita Salute San Raffaele

Carlo Signorelli

Universita Vita-Salute San Raffaele Facolta di Medicina e Chirurgia

Antonio Giampiero Russo ( $\square$ agrusso@ats-milano.it)

Agenzia di Tutela della Salute di Milano

\section{Research article}

Keywords: neoplasms, tobacco smoking, smoking, cohort studies, women's health

Posted Date: June 2nd, 2020

DOI: https://doi.org/10.21203/rs.3.rs-30973/v1

License: (c) (i) This work is licensed under a Creative Commons Attribution 4.0 International License. Read Full License 


\section{Abstract}

Background

Smoking is one of the leading causes of death worldwide, and it is strongly associated with several human cancers. However, the differential effects of cigarette smoke on the development and progression of different types of cancer remain unclear, and related data are limited.

\section{Methods}

In this longitudinal cohort study conducted among 75,324 women aged 41-76 years, we aimed to evaluate the effect of exposure to tobacco smoke on cancer development. The participants completed a questionnaire assessing sociodemographic characteristics, anthropometric measures, health status, and lifestyle habits, including smoking and dietary habits; Cox proportional hazards regression modelling was used to evaluate the association between smoking and 21 different types of cancer.

Results

After a 15-year follow-up, 9,487 cases of cancer were identified through record linkage with the Cancer Registry of Milan. Smoking was found to be positively associated with all neoplasms, with a hazard ratio 1.10 (95\% Cl, 1.04-1.16); cancer of the oral cavity 2.63 (1.72-4.01]), oesophagus 3.09 (1.37-6.96), stomach 1.52 (1.10-2.11), pancreas 1.69 (1.29-2.21), larynx 34.81 (8.07-150.14), lung 8.48 (7.09-10.14), cervix uteri 2.51 (1.38-4.57), and bladder and urinary tract 5.67 (3.96-8.14); lymphoma 1.37 (1.03-1.83); and colorectal cancer 1.30 (1.11-1.51).

Conclusions

Our results thus demonstrate how smoke exposure increases the risk of several types of cancer. Considering the increasing prevalence of smoking among women, our results highlight the need to prioritize the development of anti-smoking campaigns targeted at women in order to contrast the evident gender inequality with respect to healthcare.

\section{Background}

Despite the downward trend in the incidence of cancer over the last few years, cancer remains the second leading cause of death after cardiovascular diseases in European Union member states. Cancer accounted for $26 \%$ of all deaths in $2013^{1}$. Tobacco smoke has been recognised as an important risk factor for various human cancers and other chronic diseases, in female population, for several decades ${ }^{2-4}$ and is considered one of the largest threats to public health worldwide. According to the World Health Organization (WHO), tobacco causes more than seven million deaths each year, of which roughly six million are due to the direct use of tobacco and just below one million are associated with exposure to second-hand smoke 5 .

According to the last report of the International Agency for Research on Cancer (IARC) of 2009, a cubic centimetre of smoke has approximately $4 \times$ $10^{9}$ particles and over 5300 compounds, including monocyclic and polycyclic aromatic hydrocarbons, nitro compounds, and metals ${ }^{4,6}$. There is sufficient evidence demonstrating the carcinogenicity of at least 70 of these compounds ${ }^{6,7}$. However this is not a current evaluation and it is probably underestimated. Indeed, these data are referred to the IARC report of 2009 and therefore they are antecedent to this period.

The latest WHO report (July 2019) on the global tobacco epidemic reported that there were an estimated 1.1 billion smokers in the world, and approximately $80 \%$ of them lived in low- and middle-income countries ${ }^{9}$. In the Italian population, the prevalence of smoking among those aged greater than 14 years was $19.7 \%$ in 2017 ; the prevalence was $24.8 \%$ among men and $14.9 \%$ among women, showing a strong gender difference ${ }^{10}$. Interestingly, however, although the prevalence of smoking has been decreasing both among men and women for several decades, the mortality for cancers smoke-related is rising in women ${ }^{11}$.

Substantial evidence on the relationship between smoking and several cancers has been obtained the last few decades. Richard Doll and Bradford Hill conducted the first case-control examining the association between smoking and cancer, demonstrating a strong association between smoking and lung cancer ${ }^{2}$, and tobacco smoke has been now been recognised as the leading cause of lung cancer among smokers and those exposed to second-hand smoke ${ }^{12-15}$.

However, tobacco smoke is also associated with several other types of cancer ${ }^{1}$. Studies have examined the effect of tobacco smoke on the development of other types of cancer, such as cervical ${ }^{16-18}$, bladder $^{19-20}$, and gastro-enteric cancer ${ }^{21-25}$. Nevertheless, the evidence for the association between smoking and cancers other than lung cancer is limited and weak, particularly in female population.

Considering the evidence currently available with respect to the increase in the incidence of lung cancer in women, linked to the increase in exposure to cigarette smoke mainly in the female gender, it is essential to define the associations with other sites in order to identify the sites in which, in the coming years, an increase in cases is expected. 
Therefore, the aim of this study was to evaluate the effect of exposure to tobacco smoke on the development of cancer at different sites using data from a large cohort of women in Italy and to further validate the current evidence on the relationship between smoking and lung cancer in this cohort.

\section{Methods \\ Study cohort}

For the present study, we used the prospective FRiCaM (Risk Factors for Breast Cancer, that is, "Fattori di Rischio per il Carcinoma della Mammella") cohort. This is an observational study based on data routinely collected by the Agency for Health Protection (ATS) of Milan, a public body of the Regional Health Service - Lombardy Region, whose activity includes the evaluation of health status of the population. According to the regional law (R.L. 23/2015, 11/08/2015)http://normelombardia.consiglio.regione.lombardia.it/NormeLombardia/Accessibile/main.aspx? view=showdoc\&iddoc=Ir002015081100023), ethical approval was deemed not necessary. This study is also ethically compliant with the National Law (D.Lgs. 101/2018 https://www.gazzettaufficiale.it/eli/id/2018/09/04/18G00129/sg) and the "General Authorisation to Process Personal Data for Scientific Research Purposes" (n.8 and 9/2016, referred to in the Data Protection Authority action of 13/12/ 2018 https://www.garanteprivacy.it/home/docweb/-/docweb-display/docweb/9068972) .

Briefly, all women aged 41-76 years who resided in the municipality of Milan and were invited to undergo mammographic screening between 2003 and 2007 were included in the FRiCaM cohort. Details concerning the study design, recruitment, and questionnaire characteristics have been described previously ${ }^{26}$.

All participants completed a questionnaire on socio-demographic characteristics, anthropometric measures, health status, and lifestyle habits. In total, 131,246 women received a questionnaire and approximately $54 \%$ of them (71,398 women) completed it.

Although just above half of the potential candidates filled out the questionnaire, this does not configures a selection bias, but a non-differential selection, which does not alter the results. In fact, the dependence of the outcome, the occurrence of cancer, is consistent across the exposure categories.

The questionnaire was also sent, by mail, to women who did not undergo mammographic screening in order to collect information from a sample of non-screened women, and approximately $33 \%$ of non-screened women (6,652 women) responded to the questionnaire.

Among the 78,050 women who answered the questionnaire, 2,726 were excluded owing to a lack of information on smoking habits. Thus, the final analysis in the present study was conducted among 75,324 women.

\section{Cancer types examined}

During 15 years of follow-up, cases of cancer were identified through record linkage between the cohort study and the Cancer Registry of Milan. The Cancer Registry of Milan was accredited by IARC and has continuously collected all new invasive cancers from January 1999 . Starting from 2016, the Milan Municipality is part of cancer register of the Metropolitan area of Milan, included in Cancer Incidence in Five Continents - XI, covering the entire provinces of Milan for 3,176,180 inhabitants.

The different cancer types were coded using the 10th revision of the International Classification of Diseases. The following 21 types of cancer were included: oral cavity (C00-C08), oesophagus (C09), stomach (C16), colon and rectum (C17-C21), liver (C22), gallbladder (C23-24), pancreas (C25), larynx (C32), bronchus and lung (C33-34), skin (including melanoma) (C43-44), breast (C50), cervix uteri (C53), corpus uteri (C54), ovary (C56), kidney (C64), bladder and urinary tract (C65-67), brain and nervous system (C71-72), and thyroid (C73) cancer; lymphoma (C81-88); multiple myeloma (C90); and all types of leukaemia (C91-95).

\section{Statistical analysis}

Patients diagnosed with two or more primary cancers were included in the analyses for both cancer sites. Unconditional logistic regression analysis was used to assess the association between smoking habits disclosed in the questionnaire and several other covariates after adjustment for age to calculate the odds ratio (OR) and 95\% confidence interval (Cl). The chi-squared test was used to evaluate the differences in sociodemographic characteristics, anthropometric measures, health status, and lifestyle habits between non-smokers and smokers stratified according to the number of cigarette pack-years.

Observation time was calculated from the date of enrolment until the date of diagnosis of each type of cancer included in the study, date of withdrawal from the study, date of death, or the end of the study period. We also estimated the smoking-related hazard ratios (HRs) and corresponding 95\% Cls for each type of cancer using a Cox proportional hazard regression model adjusted for age and instruction level.

The correlation between smoking habits and cancer is proportional to the number of cigarettes smoked, which can be expressed in pack-years the higher the number of cigarettes smoked, the higher is the risk of developing cancer. In this study, we considered cigarette pack-years as a

Page $3 / 14$ 
categorical variable, and stratified patients into four groups (less than 10, 10-20, 20-30, and more than 30 pack-years smoked) based on increasing exposure to smoke. We compared the risk of cancer development in smokers and ex-smokers with that in non-smokers, and also further compared this risk between smokers stratified according to cigarette pack-years smoked and non-smokers. All analyses were performed using the SAS version 9.4 statistical software package (SAS institute Inc., Cary, NC, USA).

\section{Results}

Table 1 shows the detailed characteristics of the 75,324 participants included in the study, 16,144 (21.43\%) of whom were smokers and 59,180 (78.57\%) were never smokers or ex-smokers. According to our data, 56.4\% of the smokers smoked at least 20 cigarettes/day and $32.3 \%$ smoked at least 30 cigarettes/day. Therefore, most smokers in the cohort were heavy smokers ${ }^{27}$. Overall, the group exposed to tobacco smoke tended to be more educated, had a lower body mass index (BMI), and was younger than the non-exposed group. 
Table 1

Distribution of sociodemographic and individual characteristics among participants of the FRiCaM cohort study (ATS Milan 2019)

\begin{tabular}{|c|c|c|c|c|c|c|c|c|}
\hline & Smokers & $\begin{array}{l}\text { Non- } \\
\text { smokers }\end{array}$ & OR & $\begin{array}{l}<10 \\
\text { pack/year }\end{array}$ & $\begin{array}{l}10-19 \\
\text { pack/year }\end{array}$ & $\begin{array}{l}20-29 \\
\mathrm{pack} / \mathrm{year}\end{array}$ & $\begin{array}{l}\geq 30 \\
\text { pack/year }\end{array}$ & $\square^{2}$ \\
\hline \multicolumn{9}{|l|}{ Age } \\
\hline$<50$ & 1041 (6.5\%) & $\begin{array}{l}2384 \\
(4 \%)\end{array}$ & $1 *$ & $175(8 \%)$ & $\begin{array}{l}335 \\
(8.4 \%)\end{array}$ & 207 (6.1\%) & $204(4.5 \%)$ & \multirow[t]{6}{*}{$\begin{array}{l}< \\
0.0001\end{array}$} \\
\hline $50-54$ & $3496(21.6 \%)$ & $\begin{array}{l}8444 \\
(14.3 \%)\end{array}$ & 1,06 & $\begin{array}{l}568 \\
(26 \%)\end{array}$ & $\begin{array}{l}1001 \\
(25.3 \%)\end{array}$ & 701 (20.6\%) & $843(18.6 \%)$ & \\
\hline $55-59$ & $4304(26.7 \%)$ & $\begin{array}{l}12362 \\
(20.9 \%)\end{array}$ & 1,25 & $\begin{array}{l}595 \\
(27.2 \%)\end{array}$ & $\begin{array}{l}1079 \\
(27.2 \%)\end{array}$ & $868(25.5 \%)$ & $\begin{array}{l}1205 \\
(26.5 \%)\end{array}$ & \\
\hline $60-64$ & $3478(21.5 \%)$ & $\begin{array}{l}12875 \\
(21.8 \%)\end{array}$ & 1,62 & $\begin{array}{l}402 \\
(18.4 \%)\end{array}$ & $\begin{array}{l}725 \\
(18.3 \%)\end{array}$ & 797 (23.4\%) & $\begin{array}{l}1116 \\
(24.6 \%)\end{array}$ & \\
\hline $65-69$ & $2495(15.5 \%)$ & $\begin{array}{l}13879 \\
(23.4 \%)\end{array}$ & 2,43 & $\begin{array}{l}318 \\
(14.5 \%)\end{array}$ & $\begin{array}{l}530 \\
(13.4 \%)\end{array}$ & $522(15.4 \%)$ & $779(17.1 \%)$ & \\
\hline$\geq 70$ & $1330(8.2 \%)$ & $\begin{array}{l}9236 \\
(15.6 \%)\end{array}$ & 3,03 & $\begin{array}{l}129 \\
(5.9 \%)\end{array}$ & $\begin{array}{l}293 \\
(7.4 \%)\end{array}$ & $306(9 \%)$ & $395(8.7 \%)$ & \\
\hline mean & $58,2(44-75)$ & $\begin{array}{l}60,8 \\
(41-76)\end{array}$ & & & & & & \\
\hline tot & 16144 & 59180 & & $\begin{array}{l}2187 \\
(15.5 \%)\end{array}$ & $\begin{array}{l}3963 \\
(28.1)\end{array}$ & $\begin{array}{l}3401 \\
(24.2 \%)\end{array}$ & $\begin{array}{l}4542 \\
(32.2 \%)\end{array}$ & \\
\hline \multicolumn{8}{|l|}{ Year of enrollment } & \multirow{6}{*}{$\begin{array}{l}< \\
0.0001\end{array}$} \\
\hline 2003 & $3696(22.9 \%)$ & $\begin{array}{l}13866 \\
(23.4 \%)\end{array}$ & $1 *$ & $\begin{array}{l}524 \\
(24 \%)\end{array}$ & $\begin{array}{l}933 \\
(23.5 \%)\end{array}$ & $800(23.5 \%)$ & $\begin{array}{l}1014 \\
(22.3 \%)\end{array}$ & \\
\hline 2004 & 7225 (44.7\%) & $\begin{array}{l}27034 \\
(45.7 \%)\end{array}$ & 1,02 & $\begin{array}{l}1007 \\
(46 \%)\end{array}$ & $\begin{array}{l}1732 \\
(43.7 \%)\end{array}$ & $1478(43.5 \%)$ & $\begin{array}{l}2064 \\
(45.4 \%)\end{array}$ & \\
\hline 2005 & $4615(28.7 \%)$ & $\begin{array}{l}16469 \\
(27.8 \%)\end{array}$ & 1,02 & $\begin{array}{l}570 \\
(26 \%)\end{array}$ & $\begin{array}{l}1118 \\
(28.2 \%)\end{array}$ & $1007(29.6 \%)$ & 1307(28.8\%) & \\
\hline 2006 & $510(3.1 \%)$ & $\begin{array}{l}1472 \\
(2.5 \%)\end{array}$ & 1,01 & $68(3.2 \%)$ & $\begin{array}{l}152 \\
(3.9 \%)\end{array}$ & $97(2.8 \%)$ & $131(2.9 \%)$ & \\
\hline 2007 & $98(0.6 \%)$ & $\begin{array}{l}339 \\
(0.6 \%)\end{array}$ & 1,06 & $18(0.8 \%)$ & $28(0.7 \%)$ & $19(0.6 \%)$ & $26(0.6 \%)$ & \\
\hline \multicolumn{8}{|l|}{ Education ${ }^{1}$} & \multirow{5}{*}{$\begin{array}{l}<.0001 \\
0 .\end{array}$} \\
\hline primary school & $2712(16.9 \%)$ & $\begin{array}{l}12721 \\
(21.7 \%)\end{array}$ & 1,04 & $\begin{array}{l}311 \\
(14.3 \%)\end{array}$ & $\begin{array}{l}603 \\
(15.3 \%)\end{array}$ & $569(16.9 \%)$ & 717 (15.9\%) & \\
\hline Secondary school & $\begin{array}{l}10685 \\
(66.7 \%)\end{array}$ & $\begin{array}{l}37124 \\
(63.2 \%)\end{array}$ & 0,92 & $\begin{array}{l}1361 \\
(62.4 \%)\end{array}$ & $\begin{array}{l}2631 \\
(66.9 \%)\end{array}$ & $\begin{array}{l}2265 \\
(66.9 \%)\end{array}$ & $\begin{array}{l}3123 \\
(69.4 \%)\end{array}$ & \\
\hline University & $2629(16.4 \%)$ & $\begin{array}{l}8870 \\
(15.1 \%)\end{array}$ & $1 *$ & $\begin{array}{l}509 \\
(23.3 \%)\end{array}$ & $\begin{array}{l}702 \\
(17.8 \%)\end{array}$ & $549(16.2 \%)$ & $663(14.7 \%)$ & \\
\hline \multicolumn{8}{|l|}{ Work Activity } & \\
\hline Manager/Professional/Teacher & $3228(20.7 \%)$ & $\begin{array}{l}10941 \\
(19.1 \%)\end{array}$ & $1 *$ & $\begin{array}{l}564(26 \\
.5 \%)\end{array}$ & $\begin{array}{l}849 \\
(22 \%)\end{array}$ & $673(20.4 \%)$ & $878(19.9 \%)$ & \\
\hline Employee/merchant/artisan & $6827(43.7 \%)$ & $\begin{array}{l}22370 \\
(39 \%)\end{array}$ & 0,90 & $\begin{array}{l}903(42 \\
.4 \%)\end{array}$ & $\begin{array}{l}1673(43 \\
.4 \%)\end{array}$ & $\begin{array}{l}1444(43 \\
.7 \%)\end{array}$ & $\begin{array}{l}2024(45 \\
.8 \%)\end{array}$ & \multirow[t]{5}{*}{$<.0001$} \\
\hline Clerk/technician & $670(4.3 \%)$ & $\begin{array}{l}2298 \\
(4 \%)\end{array}$ & 0,87 & $79(3.7 \%)$ & $\begin{array}{l}200(5 \\
.2 \%)\end{array}$ & $123(3.7 \%)$ & $178(4 \%)$ & \\
\hline Skilled worker & $747(4.8 \%)$ & $\begin{array}{l}2987 \\
(5.2 \%)\end{array}$ & 0,96 & $88(4.1 \%)$ & $\begin{array}{l}171(4 \\
.4 \%)\end{array}$ & $165(5 \%)$ & $202(4.6 \%)$ & \\
\hline Worker & $1068(6.8 \%)$ & $\begin{array}{l}4218 \\
(7.3 \%)\end{array}$ & 0,98 & $\begin{array}{l}103(4 \\
.8 \%)\end{array}$ & $\begin{array}{l}242(6 \\
.3 \%)\end{array}$ & $223(6.7 \%)$ & $285(6.5 \%)$ & \\
\hline Howsewife & $2979(19.1 \%)$ & $\begin{array}{l}14178 \\
(24.7 \%)\end{array}$ & 1,13 & $\begin{array}{l}380(17 \\
.9 \%)\end{array}$ & $\begin{array}{l}696(18 \\
.1 \%)\end{array}$ & $655(19.9 \%)$ & $816(18.5 \%)$ & \\
\hline
\end{tabular}




\begin{tabular}{|c|c|c|c|c|c|c|c|c|}
\hline & Smokers & $\begin{array}{l}\text { Non- } \\
\text { smokers }\end{array}$ & OR & $\begin{array}{l}<10 \\
\text { pack/year }\end{array}$ & $\begin{array}{l}10-19 \\
\text { pack/year }\end{array}$ & $\begin{array}{l}20-29 \\
\text { pack/year }\end{array}$ & $\begin{array}{l}\geq 30 \\
\text { pack/year }\end{array}$ & $\square^{2}$ \\
\hline Never worked & $99(0.6 \%)$ & $\begin{array}{l}404 \\
(0.7 \%)\end{array}$ & 0,94 & $12(0.6 \%)$ & $22(0.6 \%)$ & $19(0.6 \%)$ & $33(0.7 \%)$ & \\
\hline \multicolumn{8}{|l|}{ Marital status ${ }^{1}$} & \multirow[t]{5}{*}{$<.0001$} \\
\hline Married/cohabitant & $9882(63 \%)$ & $\begin{array}{l}41048 \\
(71.3 \%)\end{array}$ & $1^{\star}$ & $\begin{array}{l}1427 \\
(66,8 \%)\end{array}$ & $\begin{array}{l}2600 \\
(67,2 \%)\end{array}$ & $\begin{array}{l}2110 \\
(63,6 \%)\end{array}$ & $\begin{array}{l}2558 \\
(57,9 \%)\end{array}$ & \\
\hline Separated/divorced & $1587(10.1 \%)$ & $\begin{array}{l}4652 \\
(8.1 \%)\end{array}$ & 0,70 & $192(9 \%)$ & $\begin{array}{l}333 \\
(8,6 \%)\end{array}$ & $356(10,7 \%)$ & $514(11,6 \%)$ & \\
\hline Widow & 2344 (14.9\%) & $\begin{array}{l}4230 \\
(7.4 \%)\end{array}$ & 0,47 & $\begin{array}{l}289 \\
(13,5 \%)\end{array}$ & $\begin{array}{l}522 \\
(13,6 \%)\end{array}$ & $471(14,2 \%)$ & $774(17,5 \%)$ & \\
\hline Never married & $1885(12 \%)$ & $\begin{array}{l}7629 \\
(13.2 \%)\end{array}$ & 0,73 & $\begin{array}{l}229 \\
(10,7 \%)\end{array}$ & $\begin{array}{l}411 \\
(10,6 \%)\end{array}$ & $383(11,5 \%)$ & $574(13 \%)$ & \\
\hline \multicolumn{8}{|l|}{ Age at menarche (years) ${ }^{2}$} & \multirow[t]{4}{*}{$<.0001$} \\
\hline$\leq 11$ & $4258(27 \%)$ & $\begin{array}{l}13674 \\
(23.7 \%)\end{array}$ & $1^{*}$ & $\begin{array}{l}528 \\
(24,5 \%)\end{array}$ & $\begin{array}{l}1049 \\
(26,9 \%)\end{array}$ & $909(27,4 \%)$ & $1238(28 \%)$ & \\
\hline $12-13$ & 7728 (49\%) & $\begin{array}{l}28791 \\
(49.8 \%)\end{array}$ & 1,12 & $\begin{array}{l}1135 \\
(52,8 \%)\end{array}$ & $\begin{array}{l}1911 \\
(49 \%)\end{array}$ & $1627(49 \%)$ & $\begin{array}{l}2133 \\
(48,2 \%)\end{array}$ & \\
\hline$\geq 14$ & $3788(24 \%)$ & $\begin{array}{l}15288 \\
(26.5 \%)\end{array}$ & 1,10 & $\begin{array}{l}489 \\
(22,7 \%)\end{array}$ & $\begin{array}{l}941 \\
(24,1 \%)\end{array}$ & $783(23,6 \%)$ & $\begin{array}{l}1055 \\
(23,8 \%)\end{array}$ & \\
\hline \multicolumn{8}{|l|}{ Age at first live birth (years) ${ }^{2}$} & \multirow[t]{6}{*}{$<.0001$} \\
\hline nulliparous & $2924(18,7 \%)$ & $\begin{array}{l}8762 \\
(15,3 \%)\end{array}$ & & & & & & \\
\hline$\leq 20$ & $1462(9,4 \%)$ & $\begin{array}{l}3632 \\
(6,3 \%)\end{array}$ & $1^{*}$ & $\begin{array}{l}140 \\
(8.1 \%)\end{array}$ & $\begin{array}{l}318 \\
(10 \%)\end{array}$ & $282(10.6 \%)$ & $478(13.8 \%)$ & \\
\hline $21-24$ & $3674(23,6 \%)$ & $\begin{array}{l}13394 \\
23,3 \%)\end{array}$ & 1,36 & $\begin{array}{l}445 \\
(25.7 \%)\end{array}$ & $\begin{array}{l}937 \\
(29.5 \%)\end{array}$ & $737(27.9 \%)$ & $\begin{array}{l}1018 \\
(29.3 \%)\end{array}$ & \\
\hline $25-29$ & $4798(30,8 \%)$ & $\begin{array}{l}20661 \\
(36 \%)\end{array}$ & 1,51 & $\begin{array}{l}736 \\
(42.4 \%)\end{array}$ & $\begin{array}{l}1205 \\
(37.9 \%)\end{array}$ & $\begin{array}{l}1080 \\
(40.8 \%)\end{array}$ & $\begin{array}{l}1246 \\
(35.9 \%)\end{array}$ & \\
\hline$\geq 30$ & $2737(17,5 \%)$ & $\begin{array}{l}10962 \\
(19,1 \%)\end{array}$ & 1,46 & $\begin{array}{l}413 \\
(23.8 \%)\end{array}$ & $\begin{array}{l}716 \\
(22.6 \%)\end{array}$ & $547(20.7 \%)$ & $729(21 \%)$ & \\
\hline \multicolumn{8}{|l|}{ Menopausal status } & \multirow[t]{3}{*}{$<.0001$} \\
\hline Premenopause & $2771(18.5 \%)$ & $\begin{array}{l}8227 \\
(15.2 \%)\end{array}$ & $1^{*}$ & $\begin{array}{l}516 \\
(24.9 \%)\end{array}$ & $\begin{array}{l}821 \\
(22.2 \%)\end{array}$ & $565(17.7 \%)$ & $571(13.4 \%)$ & \\
\hline Postmenopause & $12228(81.5 \%)$ & $\begin{array}{l}45983 \\
(84.8 \%)\end{array}$ & 0,73 & $\begin{array}{l}1559 \\
(75.1 \%)\end{array}$ & $\begin{array}{l}2883 \\
(77.8 \%)\end{array}$ & $\begin{array}{l}2633 \\
(82.3 \%)\end{array}$ & $\begin{array}{l}3688 \\
(86.6 \%)\end{array}$ & \\
\hline \multicolumn{9}{|l|}{ BMI } \\
\hline$<18.5$ & $977(6,3 \%)$ & $\begin{array}{l}1936 \\
(3,4 \%)\end{array}$ & 0,63 & $\begin{array}{l}131 \\
(6,2 \%)\end{array}$ & $\begin{array}{l}2441 \\
(63,8 \%)\end{array}$ & $200(6,1 \%)$ & $280(6,4 \%)$ & \multirow[t]{6}{*}{$<.0001$} \\
\hline $18.5-24.9$ & $9521(61,5 \%)$ & $\begin{array}{l}30372 \\
(54 \%)\end{array}$ & $1^{\star}$ & $\begin{array}{l}1362 \\
(64,5 \%)\end{array}$ & $\begin{array}{l}242 \\
(6,3 \%)\end{array}$ & $\begin{array}{l}2063 \\
(63,1 \%)\end{array}$ & $\begin{array}{l}2535 \\
(58,2 \%)\end{array}$ & \\
\hline $25-29.9$ & $3939(25,5 \%)$ & $\begin{array}{l}18152 \\
(32,3 \%)\end{array}$ & 1,36 & $\begin{array}{l}494 \\
(23,4 \%)\end{array}$ & $\begin{array}{l}922 \\
(24 \%)\end{array}$ & $816(25 \%)$ & $\begin{array}{l}1165 \\
(26,7 \%)\end{array}$ & \\
\hline$\geq 30$ & $1036(6,7 \%)$ & $\begin{array}{l}5721 \\
(10,2 \%)\end{array}$ & 1,65 & $\begin{array}{l}125 \\
(5,9 \%)\end{array}$ & $\begin{array}{l}223 \\
(5,8 \%)\end{array}$ & $189(5,8 \%)$ & $380(8,7 \%)$ & \\
\hline mean & $\begin{array}{l}23,5(\mathrm{DS}= \\
4,1)\end{array}$ & $\begin{array}{l}24,6 \\
(\text { DS }= \\
4,4)\end{array}$ & & & & & & \\
\hline Total & 16144 & 59180 & & & & & & \\
\hline
\end{tabular}

The smokers in this study had a mean age of 58.2 years (standard deviation [SD] = 6.5 years) and a mean BMl of $23.5 \mathrm{~kg} / \mathrm{m}^{2}\left(\mathrm{SD}=4.1 \mathrm{~kg} / \mathrm{m}^{2}\right)$. The non-smokers were older, with a mean age of 60.8 year $\left(S D=6.9\right.$ years), and their mean BMl was $24.6 \mathrm{~kg} / \mathrm{m}^{2}\left(\mathrm{SD}=4.4 \mathrm{~kg} / \mathrm{m}^{2}\right)$. Moreover, 
$16.4 \%$ of the smokers went to university, $66.7 \%$ went to a secondary school, and $16.9 \%$ attended only primary school or did not attend any school at all. In contrast, only $15.1 \%$ of non-smokers completed university, $63.2 \%$ had a high school diploma, and $21.7 \%$ had completed only primary school.

With regard to marital status, $71.3 \%$ of non-smokers were married or cohabitant, whereas this rate was $63 \%$ among smokers; $10.1 \%$ of smokers and $8.1 \%$ of non-smokers were divorced. Moreover, $14.9 \%$ and $7.4 \%$ of smokers and non-smokers were widowed, respectively, and $12 \%$ and $13.2 \%$ were unmarried.

Table 2 describes the relationship between dietary and smoking habits. In general, smokers had a lower intake of vegetables, fruit, fish, cheese, and white meat than non-smokers, and they consumed more red meat. Furthermore, the per-week portion consumption of fruits and vegetables appeared to decrease with an increase in the number of cigarettes smoked.

In addition, $75.7 \%$ of non-smokers consumed more than one portion of vegetables a day, while only $68.3 \%$ of smokers consumed the same amount of vegetables; this percentage decreased to $63.9 \%$ if only heavy smokers were considered. Similarly, $89.1 \%$ of non-smokers consumed one portion of fruit a day; this rate was $76.5 \%$ among smokers and $70 \%$ among heavy smokers. Only small differences in the consumption of other foods were noted between smokers and non-smokers (Table 2). 
Table 2

Distribution of dietary habits among participants of the FRiCaM cohort study (ATS Milan 2019)

\begin{tabular}{|c|c|c|c|c|c|c|c|c|}
\hline $\begin{array}{l}\text { One portion of } \\
\text { vegetables }\end{array}$ & Smokers & $\begin{array}{l}\text { Non- } \\
\text { smokers }\end{array}$ & OR & $\begin{array}{l}<10 \\
\text { pack/year }\end{array}$ & $\begin{array}{l}10-19 \\
\text { pack/year }\end{array}$ & $\begin{array}{l}20-29 \\
\text { pack/year }\end{array}$ & $\begin{array}{l}\geq 30 \\
\text { pack/year }\end{array}$ & $x^{2}$ \\
\hline $\begin{array}{l}\text { Less than once a } \\
\text { week }\end{array}$ & $385(2,4 \%)$ & $797(1,4 \%)$ & 0.50 & $31(1,4 \%)$ & $60(1,5 \%)$ & $67(2 \%)$ & $165(3,7 \%)$ & $<, 0001$ \\
\hline 1 to 6 times a week & $4609(29,3 \%)$ & $\begin{array}{l}13237 \\
(22,9 \%)\end{array}$ & 0.71 & $520(24,4 \%)$ & $1022(26,3 \%)$ & $995(29,7 \%)$ & $1449(32,4 \%)$ & \\
\hline Once a day or more & $10763(68,3 \%)$ & $\begin{array}{l}43801 \\
(75,7 \%)\end{array}$ & $1 *$ & $\begin{array}{l}1582 \\
(74,2 \%)\end{array}$ & $2804(72,2 \%)$ & $2289(68,3 \%)$ & $2854(63,9 \%)$ & \\
\hline One portion of fruit & & & & & & & & $<, 0001$ \\
\hline $\begin{array}{l}\text { Less than once a } \\
\text { week }\end{array}$ & $660(4,2 \%)$ & $661(1,1 \%)$ & 0.48 & $41(1,9 \%)$ & $112(2,9 \%)$ & $138(4,2 \%)$ & $296(6,7 \%)$ & \\
\hline 1 to 6 times a week & $3013(19,3 \%)$ & $5644(9,8 \%)$ & 0.73 & $348(16,3 \%)$ & $683(17,7 \%)$ & $615(18,6 \%)$ & $1023(23,3 \%)$ & \\
\hline Once a day or more & $\begin{array}{l}11925 \\
(76,5 \%)\end{array}$ & $\begin{array}{l}51287 \\
(89,1 \%)\end{array}$ & $1 *$ & $\begin{array}{l}1751 \\
(81,8 \%)\end{array}$ & $3060(79,4 \%)$ & $2560(77,2 \%)$ & 3078 (70\%) & \\
\hline One portion of cheese & & & & & & & & $<, 0001$ \\
\hline $\begin{array}{l}\text { Less than once a } \\
\text { week }\end{array}$ & $1294(8,4 \%)$ & $3868(6,9 \%)$ & $1 *$ & $146(6,9 \%)$ & $296(7,8 \%)$ & $253(7,8 \%)$ & $437(10 \%)$ & \\
\hline 1 to 6 times a week & $11003(71,7 \%)$ & $\begin{array}{l}39958 \\
(70,8 \%)\end{array}$ & 1.31 & $\begin{array}{l}1546 \\
(73,6 \%)\end{array}$ & $2774(73,2 \%)$ & $2364(72,7 \%)$ & $3029(69,5 \%)$ & \\
\hline Once a day or more & $3047(19,9 \%)$ & $\begin{array}{l}12581 \\
(22,3 \%)\end{array}$ & 1.96 & $410(19,5 \%)$ & $720(19 \%)$ & $635(19,5 \%)$ & $891(20,5 \%)$ & \\
\hline $\begin{array}{l}\text { One portion of red } \\
\text { meat }\end{array}$ & & & & & & & & $<, 0001$ \\
\hline $\begin{array}{l}\text { Less than once a } \\
\text { week }\end{array}$ & $3823(24,7 \%)$ & $\begin{array}{l}14490 \\
(25,6 \%)\end{array}$ & $1 *$ & $492(23,3 \%)$ & $913(23,8 \%)$ & $813(24,8 \%)$ & $1165(26,5 \%)$ & \\
\hline 1 to 6 times a week & $\begin{array}{l}11111 \\
(71,9 \%)\end{array}$ & $\begin{array}{l}40481 \\
(71,4 \%)\end{array}$ & 1.12 & $\begin{array}{l}1558 \\
(73,8 \%)\end{array}$ & $2825(73,7 \%)$ & $2384(72,7 \%)$ & $3026(69 \%)$ & \\
\hline Once a day or more & $532(3,4 \%)$ & 1705 (3\%) & 1.64 & $62(2,9 \%)$ & $95(2,5 \%)$ & $82(2,5 \%)$ & $196(4,5 \%)$ & \\
\hline $\begin{array}{l}\text { One portion of white } \\
\text { meat }\end{array}$ & & & & & & & & $<, 0001$ \\
\hline $\begin{array}{l}\text { Less than once a } \\
\text { week }\end{array}$ & $2732(17,7 \%)$ & $\begin{array}{l}7233 \\
(12,7 \%)\end{array}$ & 0.52 & $314(14,9 \%)$ & $620(16,2 \%)$ & $547(16,5 \%)$ & $935(21,4 \%)$ & \\
\hline 1 to 6 times a week & $\begin{array}{l}12236 \\
(79,2 \%)\end{array}$ & $\begin{array}{l}47686 \\
(83,5 \%)\end{array}$ & 0.66 & $\begin{array}{l}1729 \\
(82,2 \%)\end{array}$ & $3099(81,1 \%)$ & $2664(80,6 \%)$ & $3285(75,3 \%)$ & \\
\hline Once a day or more & $487(3,1 \%)$ & $2165(3,8 \%)$ & $1 *$ & $61(2,9 \%)$ & $103(2,7 \%)$ & $95(2,9 \%)$ & $140(3,3 \%)$ & \\
\hline One portion of fish & & & & & & & & $<, 0001$ \\
\hline $\begin{array}{l}\text { Less than once a } \\
\text { week }\end{array}$ & 5330 (34\%) & $\begin{array}{l}16282 \\
(28,2 \%)\end{array}$ & 0.53 & $627(29,3 \%)$ & $1202(31,1 \%)$ & $1155(34,6 \%)$ & $1722(38,8 \%)$ & \\
\hline 1 to 6 times a week & 10195 (65\%) & $\begin{array}{l}40747 \\
(70,6 \%)\end{array}$ & 0.69 & $\begin{array}{l}1492 \\
(69,6 \%)\end{array}$ & $2633(67,9 \%)$ & $2157(64,7 \%)$ & $2674(60,2 \%)$ & \\
\hline Once a day or more & $167(1 \%)$ & $691(1,2 \%)$ & $1 *$ & $23(1,1 \%)$ & $40(1 \%)$ & $23(0,7 \%)$ & $46(1 \%)$ & \\
\hline
\end{tabular}

The risk estimates for the association between cigarette smoking and cancer are illustrated in Table 3. Cancer was found to have developed in 9,487 cases, representing $12.2 \%$ of the cohort. 
Table 3

Hazard ratio and corresponding confidence interval for smoker vs non-smoker (ATS Milan 2019).

\begin{tabular}{|c|c|c|c|c|c|c|c|c|c|c|c|}
\hline & Number & $\begin{array}{l}\text { Inciden } \\
(* 1000\end{array}$ & & & Smoker & & Pack-Ye & & & & yuare for \\
\hline & $\begin{array}{l}\text { of } \\
\text { cases }\end{array}$ & Exp & $\begin{array}{l}\text { Non } \\
\text { Exp }\end{array}$ & No & Ex & Yes & $<$ & & $20-29$ & & \\
\hline Oral cavity & 112 & 1.97 & 0.85 & 1* & 1,10 & 2.63 & 1.31 & 2.21 & 1.71 & 4.88 & 0.19 \\
\hline & & & & & $\begin{array}{l}(0.65- \\
1.87)\end{array}$ & $\begin{array}{l}(1.72- \\
4.01)\end{array}$ & $\begin{array}{l}(0.40- \\
4.23)\end{array}$ & $\begin{array}{l}(1.07- \\
4.54)\end{array}$ & $\begin{array}{l}(0.73- \\
4.01)\end{array}$ & $\begin{array}{l}(2.94- \\
8.11)\end{array}$ & \\
\hline Oesophagus & 33 & 0.59 & 0.25 & $1 *$ & 1.41 & 3.09 & 0 & 1.13 & 3.53 & 5.90 & 0.26 \\
\hline & & & & & $\begin{array}{l}(0.55- \\
3.59)\end{array}$ & $\begin{array}{l}(1.37- \\
6.96)\end{array}$ & $(0-)$ & $\begin{array}{l}(0.15- \\
8.74)\end{array}$ & $\begin{array}{l}(1.00- \\
12.51)\end{array}$ & $\begin{array}{l}(2.33- \\
14.96)\end{array}$ & \\
\hline Stomach & 225 & 2.48 & 2.105 & $1 *$ & 1.03 & 1.52 & 0.84 & 1.17 & 1.39 & 2.08 & 0.46 \\
\hline & & & & & $\begin{array}{l}(0.72- \\
1.46)\end{array}$ & $\begin{array}{l}(1.10- \\
2.11)\end{array}$ & $\begin{array}{l}(0.31- \\
2.29)\end{array}$ & $\begin{array}{l}(0.61- \\
2.24)\end{array}$ & $\begin{array}{l}(0.75- \\
2.59)\end{array}$ & $\begin{array}{l}(1.32- \\
3.28)\end{array}$ & \\
\hline Colon \& Rectum & 1080 & 11.03 & 10.34 & $1 *$ & 1.16 & 1.30 & 1.56 & 1.30 & 1.33 & 1.13 & 0.015 \\
\hline & & & & & $\begin{array}{l}(1.00- \\
1.35)\end{array}$ & $\begin{array}{l}(1.11- \\
1.51)\end{array}$ & $\begin{array}{l}(1.12- \\
2.18)\end{array}$ & $\begin{array}{l}(0.99- \\
1.71)\end{array}$ & $\begin{array}{l}(1.00- \\
1.76)\end{array}$ & $\begin{array}{l}(0.86- \\
1.47)\end{array}$ & \\
\hline Liver & 143 & 1.33 & 1.403 & $1 *$ & 0.84 & 1.15 & n.a.** & 1.14 & 1.60 & 0.80 & 0.76 \\
\hline & & & & & $\begin{array}{l}(0.54- \\
1.30)\end{array}$ & $\begin{array}{l}(0.75- \\
1.76)\end{array}$ & & $\begin{array}{l}(0.53- \\
2.48)\end{array}$ & $\begin{array}{l}(0.80- \\
3.18)\end{array}$ & $\begin{array}{l}(0.35- \\
1.84)\end{array}$ & \\
\hline Gallbladder & 114 & 1.33 & 1.05 & $1 *$ & 1.17 & 1.54 & 1.19 & 1.78 & 0.99 & 2.05 & 0.33 \\
\hline & & & & & $\begin{array}{l}(0.73- \\
1.87)\end{array}$ & $\begin{array}{l}(0.97- \\
2.43)\end{array}$ & $\begin{array}{l}(0.37- \\
3.82)\end{array}$ & $\begin{array}{l}(0.84- \\
3.76)\end{array}$ & $\begin{array}{l}(0.36- \\
2.73)\end{array}$ & $\begin{array}{l}(1.07- \\
3.91)\end{array}$ & \\
\hline Pancreas & 309 & 3.77 & 2.79 & $1 *$ & 1.18 & 1.69 & 1.39 & 1.50 & 1.56 & 1.93 & 0.067 \\
\hline & & & & & $\begin{array}{l}(0.89- \\
1.58)\end{array}$ & $\begin{array}{l}(1.29- \\
2.21)\end{array}$ & $\begin{array}{l}(0.71- \\
2.73)\end{array}$ & $\begin{array}{l}(0.92- \\
2.46)\end{array}$ & $\begin{array}{l}(0.94- \\
2.58)\end{array}$ & $\begin{array}{l}(1.29- \\
2.89)\end{array}$ & \\
\hline Larynx & 29 & 0.965 & 0.098 & $1 *$ & 8.31 & 34.81 & 12.37 & 13.51 & 18.79 & 22.76 & 0.0019 \\
\hline & & & & & $\begin{array}{l}(1.66- \\
41.43)\end{array}$ & $\begin{array}{l}(8.07- \\
150.14)\end{array}$ & $\begin{array}{l}(2.22- \\
68.92)\end{array}$ & $\begin{array}{l}(3.31- \\
55.12)\end{array}$ & $\begin{array}{l}(4.98- \\
70.98)\end{array}$ & $\begin{array}{l}(6.75- \\
76.80)\end{array}$ & \\
\hline Bronchus and lung & 829 & 21.14 & 4.54 & $1 *$ & 3.07 & 8.48 & 1.17 & 3.73 & 5.90 & 12.03 & $<.0001$ \\
\hline & & & & & $\begin{array}{l}(2.49- \\
3.78)\end{array}$ & $\begin{array}{l}(7.09- \\
10.14)\end{array}$ & $\begin{array}{l}(0.64- \\
2.15)\end{array}$ & $\begin{array}{l}(2.81- \\
4.95)\end{array}$ & $\begin{array}{l}(4.60- \\
7.57)\end{array}$ & $\begin{array}{l}(9.99- \\
14.49)\end{array}$ & \\
\hline $\begin{array}{l}\text { Skin cancers (including } \\
\text { melanoma) }\end{array}$ & 1429 & 11.48 & 14.51 & $1 *$ & 1.07 & & & & 1.04 & 0.85 & 0.47 \\
\hline & & & & & $\begin{array}{l}(0.94- \\
1.22)\end{array}$ & $\begin{array}{l}(0.78- \\
1.04)\end{array}$ & $\begin{array}{l}(0.53- \\
1.13)\end{array}$ & $\begin{array}{l}(0.81- \\
1.33)\end{array}$ & $\begin{array}{l}(0.80- \\
1.35)\end{array}$ & $\begin{array}{l}(0.66- \\
1.09)\end{array}$ & \\
\hline Breast & 2952 & 28.21 & 28.77 & $1 *$ & 0.91 & 0.96 & 0.97 & 0.88 & 1.12 & 0.99 & 0.036 \\
\hline & & & & & $\begin{array}{l}(0.83- \\
0.99)\end{array}$ & $\begin{array}{l}(0.87- \\
1.05)\end{array}$ & $\begin{array}{l}(0.98- \\
0.78)\end{array}$ & $\begin{array}{l}(0.74- \\
1.05)\end{array}$ & $\begin{array}{l}(0.95- \\
1.33)\end{array}$ & $\begin{array}{l}(0.85- \\
1.16)\end{array}$ & \\
\hline Cervix uteri & 62 & 0.965 & 0.504 & $1 *$ & 1.91 & 2.51 & 1.71 & 2.76 & 2.70 & 2.48 & 0.019 \\
\hline & & & & & $\begin{array}{l}(1.02- \\
3.59)\end{array}$ & $\begin{array}{l}(1.38- \\
4.57)\end{array}$ & $\begin{array}{l}(0.40- \\
7.28)\end{array}$ & $\begin{array}{l}(1.12- \\
6.81)\end{array}$ & $\begin{array}{l}(1.03- \\
7.11)\end{array}$ & $\begin{array}{l}(1.01- \\
6.08)\end{array}$ & \\
\hline Corpus uteri & 428 & 3.77 & 4.26 & $1 *$ & 0.75 & 0.82 & 0.74 & 0.91 & 0.72 & 0.98 & 0.015 \\
\hline & & & & & $\begin{array}{l}(0.58- \\
0.96)\end{array}$ & $\begin{array}{l}(0.64- \\
1.06)\end{array}$ & $\begin{array}{l}(0.39- \\
1.40)\end{array}$ & $\begin{array}{l}(0.58- \\
1.40)\end{array}$ & $\begin{array}{l}(0.43- \\
1.21)\end{array}$ & $\begin{array}{l}(0.66- \\
1.46)\end{array}$ & \\
\hline Ovary & 273 & 2.297 & 2.74 & $1 *$ & 1.04 & 0.88 & 1.12 & 0.83 & 0.98 & 0.43 & 0.91 \\
\hline & & & & & $\begin{array}{l}(0.78- \\
1.34)\end{array}$ & $\begin{array}{l}(0.64- \\
1.21)\end{array}$ & $\begin{array}{l}(0.58- \\
2.19)\end{array}$ & $\begin{array}{l}(0.46- \\
1.49)\end{array}$ & $\begin{array}{l}(0.54- \\
1.75)\end{array}$ & $\begin{array}{l}(0.20- \\
0.92)\end{array}$ & \\
\hline
\end{tabular}




\begin{tabular}{|c|c|c|c|c|c|c|c|c|c|c|c|}
\hline \multirow[b]{2}{*}{ Kidney } & \multirow{2}{*}{$\begin{array}{l}\text { Number } \\
167\end{array}$} & \multicolumn{2}{|c|}{$\begin{array}{l}\text { Incidence } \\
(\star 10000)\end{array}$} & \multirow[b]{2}{*}{$1 *$} & \multicolumn{2}{|l|}{ Smoker } & \multicolumn{2}{|c|}{ Pack-Years } & \multirow[b]{2}{*}{1.02} & \multicolumn{2}{|c|}{$\begin{array}{l}\text { Chi-square for } \\
\text { trend }\end{array}$} \\
\hline & & 1.56 & 1.64 & & 1.10 & 1.11 & 1.41 & 0.39 & & 1.41 & 0.58 \\
\hline & & & & & $\begin{array}{l}(0.75- \\
1.60)\end{array}$ & $\begin{array}{l}(0.74- \\
1.65)\end{array}$ & $\begin{array}{l}(0.62- \\
3.25)\end{array}$ & $\begin{array}{l}(0.12- \\
1.24)\end{array}$ & $\begin{array}{l}(0.47- \\
2.12)\end{array}$ & $\begin{array}{l}(0.79- \\
2.52)\end{array}$ & \\
\hline \multirow[t]{2}{*}{ Bladder. urinary tract } & 170 & 3.63 & 1.12 & $1 *$ & 2.37 & 5.67 & 2.53 & 3.06 & 6.87 & 7.40 & $<.0001$ \\
\hline & & & & & $\begin{array}{l}(1.56- \\
3.60)\end{array}$ & $\begin{array}{l}(3.96- \\
8.14)\end{array}$ & $\begin{array}{l}(1.01- \\
6.35)\end{array}$ & $\begin{array}{l}(1.59- \\
5.87)\end{array}$ & $\begin{array}{l}(4.21- \\
11.21)\end{array}$ & $\begin{array}{l}(4.79-11- \\
45)\end{array}$ & \\
\hline \multirow{2}{*}{$\begin{array}{l}\text { Brain and nervous } \\
\text { system }\end{array}$} & 123 & 1.485 & 1.21 & $1^{*}$ & 1.44 & 1.18 & 1.12 & 1.26 & 1.84 & 0.69 & 0.09 \\
\hline & & & & & $\begin{array}{l}(0.94- \\
2.21)\end{array}$ & $\begin{array}{l}(0.74- \\
1.91)\end{array}$ & $\begin{array}{l}(0.35- \\
3.58)\end{array}$ & $\begin{array}{l}(0.54- \\
2.92)\end{array}$ & $\begin{array}{l}(0.88- \\
3.86)\end{array}$ & $\begin{array}{l}(0.25- \\
1.90)\end{array}$ & \\
\hline \multirow[t]{2}{*}{ Thyroid } & 159 & 1.56 & 1.54 & $1^{*}$ & 0.89 & 0.92 & 1.12 & 0.95 & 0.76 & 0.68 & 0.53 \\
\hline & & & & & $\begin{array}{l}(0.60- \\
1.32)\end{array}$ & $\begin{array}{l}(0.61- \\
1.37)\end{array}$ & $\begin{array}{l}(0.48- \\
2.56)\end{array}$ & $\begin{array}{l}(0.47- \\
1.89)\end{array}$ & $\begin{array}{l}(0.33- \\
1.73)\end{array}$ & $\begin{array}{l}(0.32- \\
1.47)\end{array}$ & \\
\hline \multirow[t]{2}{*}{ Lymphomas } & 298 & 3.26 & 2.79 & $1 *$ & 1.20 & 1.37 & 1.57 & 1.30 & 1.62 & 1.08 & 0.12 \\
\hline & & & & & $\begin{array}{l}(0.90- \\
1.59)\end{array}$ & $\begin{array}{l}(1.03- \\
1.83)\end{array}$ & $\begin{array}{l}(0.85- \\
2.90)\end{array}$ & $\begin{array}{l}(0.77- \\
2.18)\end{array}$ & $\begin{array}{l}(0.99- \\
2.65)\end{array}$ & $\begin{array}{l}(0.64- \\
1.81)\end{array}$ & \\
\hline \multirow[t]{2}{*}{ Multiple myeloma } & 115 & 1.103 & 1.12 & $1^{*}$ & 1.02 & 1.15 & 1.36 & 1.52 & 0.89 & 1.20 & 0.86 \\
\hline & & & & & $\begin{array}{l}(0.64- \\
1.62)\end{array}$ & $\begin{array}{l}(0.71- \\
1.85)\end{array}$ & $\begin{array}{l}0.49- \\
3.77)\end{array}$ & $\begin{array}{l}(0.72- \\
3.21)\end{array}$ & $\begin{array}{l}(0.32- \\
2.45)\end{array}$ & $\begin{array}{l}(0.55- \\
2.62)\end{array}$ & \\
\hline \multirow[t]{2}{*}{ All leukaemia } & 128 & 0.919 & 1.33 & $1^{*}$ & 1.22 & 0.85 & 0.63 & 1.42 & 0.20 & 0.74 & 0.43 \\
\hline & & & & & $\begin{array}{l}(0.81- \\
1.85)\end{array}$ & $\begin{array}{l}(0.52- \\
1.41)\end{array}$ & $\begin{array}{l}(0.15- \\
2.60)\end{array}$ & $\begin{array}{l}(0.68- \\
2.97)\end{array}$ & $\begin{array}{l}(0.03- \\
1.42)\end{array}$ & $\begin{array}{l}(0.30- \\
1.84)\end{array}$ & \\
\hline \multirow[t]{2}{*}{ All neoplasms } & 9487 & 112.09 & 90.96 & $1^{*}$ & 1.38 & 1.10 & 1.07 & 1.19 & 1.41 & 1.63 & $<.0001$ \\
\hline & & & & & $\begin{array}{l}(1.31- \\
1.45)\end{array}$ & $\begin{array}{l}(1.04- \\
1.16)\end{array}$ & $\begin{array}{l}(0.94- \\
1.21)\end{array}$ & $\begin{array}{l}(1.09- \\
1.31)\end{array}$ & $\begin{array}{l}(1.29- \\
1.55)\end{array}$ & $\begin{array}{l}(1.51- \\
1.76)\end{array}$ & \\
\hline
\end{tabular}

Overall, the person-time incidence for all cancers included was 112.09 person-years for every 10,000 subjects among smokers and 90.96 personyears for every 10,000 subjects among smokers and ex-smokers. A positive association was found between smoking and all types of cancers, with an $\mathrm{HR}=1.10\left(\mathbb{Z}^{2}<0001\right)$ for smokers and $\mathrm{HR}=1.38$ in ex-smokers. The risk increased with the number of cigarettes smoked, from 1.07 (Cl: $0.94-$ 1.21) among those who smoked less than 10 pack-years to 1.63 (Cl: 1.51-1.76) among those who smoked more than 30 pack-years.

Smoke exposure was positively associated with several types of cancer, as follows: colorectal ( $H R=1.30$ among smokers and $H R=1.16$ among ex-smokers, $\left.\mathbb{\bigotimes}^{2}=.0015\right)$, laryngeal $\left(H R=34.81\right.$ among smokers and 8.31 among ex-smokers, $\left.\mathbb{\bigotimes}^{2}=.0019\right)$, lung $(H R=8.48$ among smokers and 3.07 among ex-smokers, $\left.\mathbb{\bigotimes}^{2}<0001\right)$, cervical $\left(H R=2.51\right.$ among smokers and $H R=1.91$ among ex-smokers, $\left.\mathbb{\bigotimes}^{2}=0.019\right)$, and bladder cancer $(H R=5.67$ among smokers and $H R=2.37$ among ex-smokers, $\mathbb{Q}^{2}<0001$ ). A negative correlation was found for uterine and breast cancer, with an $H R=0.82$ for uterine cancer among smokers and an $\mathrm{HR}=0.75$ among ex-smokers $\left(\mathbb{\nabla}^{2}=0.015\right)$, and an $\mathrm{HR}=0.96$ for breast cancer among smokers and an $\mathrm{HR}=$ 0.91 among ex-smokers ( $\left.\mathbb{\triangle}^{2}=0.036\right)$. For cancer of the oral cavity (HR $\left.2.63[95 \% \mathrm{Cl} 1.72-4.01]\right)$, oesophagus (3.09 [1.37-6.96]), stomach (1.52 [1.10-2.11]), and pancreas (1.69 [1.29-2.21]) and for lymphomas (1.37 [1.03-1.83]), current smoking status was associated with cancer development, without any trend effect (Table 2).

The risk of cancer increased with the number of cigarettes. In more detail, for laryngeal cancer, the risk increased from HR=12.37 (Cl: 2.22-68.92), for those who smoked less than 10 pack/year to $H R=22.76$ (Cl: $6.75-76.80)$ for heavy smokers; for lung cancer, from $\mathrm{HR}=1.17(\mathrm{Cl}: 0.64-2.15)$ to $\mathrm{HR}=12.03$ (Cl: 9.99-14.49); for cervical cancer, from HR = 1.91 (Cl: 0.41-7.28) to 2.48 (Cl: 1.01-6.08); and for bladder cancer, from HR = 2.53 (Cl: 1.01-6.35) to $\mathrm{HR}=7.40$ (Cl: 4.79-11.45). In contrast, colorectal cancer bucked the trend, for we observed a decrease with the number of cigarettes smoked, from HR $=1.56$ (Cl: $1.12-2.18)$ for people that smoked less than 10 pack/years of cigarettes to HR $=1.13$ (Cl: $0.86-1.47$ ) for people that smoked more than 40 pack/year (Table 2).

\section{Discussion}

In thus study, which aimed to analyse the association between cigarette smoke and the development of cancer of different types in women, we evaluated a cohort of 75,324 female residents from the municipality of Milan who underwent mammographic screening. Through linkage with the Cancer Registry of Milan, we found that 9,487 (12.6\%) women from the cohort were diagnosed with cancer after enrolment. The present study 
showed that smoking was positively associated with an increased risk of several types of cancer, including tracheal, bronchial and lung, laryngeal, bladder and urinary tract, cervical, and colorectal cancer.

For colorectal cancer, the HR was 1.30 (Cl: 1.11-1.51) in the smoker population. This association is not well demonstrated in the literature. Studies conducted before 1980 failed to identify any association between cigarette smoking and colorectal cancer. Over the following twenty years, some studies found an association between long-term exposure to smoke and colorectal cancer among heavy smokers. Giovannucci et al, ${ }^{28}$ found an association between smoking and the presence of colorectal cancer in women (relative risk $[\mathrm{RR}]=1.11 ; 95 \% \mathrm{Cl}=0.93-1.34$ ) 35 years after smoking initiation. Similar results were noted in cohort ${ }^{29-32}$ and case-control studies ${ }^{33-34}$. A more recent meta-analysis of observational studies conducted by Yang et al. ${ }^{35}$ in 2016 found an association between passive smoke exposure and rectal cancer $(\mathrm{RR}=1.14$ [95\% $\left.\mathrm{Cl}=1.05-1.24]\right)$.

The present study also found a negative association between smoking and uterine cancer, with an HR of 0.82 , decreasing to 0.75 in ex-smokers. These results are consistent with the current literature ${ }^{36-37}$.

For ovarian cancer, the HR was 0.88 in smokers and 1.04 in ex-smokers. Previous results in this regard have been conflicting, with some studies reporting a negative association and some meta-analyses reporting a positive association ${ }^{38}$. These discrepancies could be due to the heterogeneity in the aetiology of the different histotypes of ovarian cancer.

Further, the present study also confirmed an association between smoking and cancer of the lung, bronchi, trachea, larynx, bladder, and pancreas. It must be emphasized that the smokers in this cohort were largely heavy smokers, which clearly affected the results. Smokers had a higher risk of developing lung cancer ( $\mathrm{HR}=8.48$ vs. non-smokers). When we considered exposure-related stratification, we found that the risk among those smoking < 10 pack-years versus the risk among never and ex-smokers combined was 0.86 . In contrast, when the exposure was $<30$ pack-years, the risk was considerably higher at 8.81 , clearly demonstrating that heavy smokers contributed highly to the first HR of 8.48 . The HR of 0.86 noted for those consuming < 10 pack/years can be explained by the fact that this risk was measured relative to the risk among never and ex-smokers combined, and despite the reduction in the risk observed after quitting smoking, the risk among ex-smokers is still higher than that among never smokers.

This study has some limitations that must be considered, the most important of which was the low number of cases of some types of cancer, that made it difficult to look for associations with smoke. This limitation could be addressed, in future studies, by collecting more data and following the cohort for a more extended period of time.

Despite these, the study has several strengths: the cohort size, the long follow-up duration of 15 years, and the significant predominance of heavy smokers in the exposed group.

\section{Conclusions}

In conclusion, the evidence from this population-based cohort study confirms that cigarette smoke increases the overall risk of cancer and specifically raises the risk of cancer of the lungs, bronchi, trachea, larynx, colon, pancreas, and cervix. One important point of our study is that we identified an increased risk of multiple types of cancer, for which there was previously little evidence. Secondly, the present study was conducted considering the increasing cancer incidence among women due to the rising trend of smoking habits in this group. This trend is expected to expand the current gender gap in healthcare. Our results thus highlight the need to develop effective anti-tobacco health initiatives targeting women, such as campaigns for smoking reduction, mass advertising campaigns in the media, and health education at school, which if conducted properly and for extended durations, could effectively reduce the current gender inequalities in healthcare.

\section{Abbreviations}

BMI, body mass index; $\mathrm{Cl}$, confidence interval; HR, hazard ratio; OR, odds ratio; RR, relative risk; SD, standard deviation; WHO, World Health Organization.

\section{Declarations}

\section{Ethics approval and consent to participate}

This is an observational study based on data routinely collected by the Agency for Health Protection (ATS) of Milan, a public body of the Regional Health Service - Lombardy Region. The ATS has among its institutional functions, the government of the care pathway at the individual level in the regional social and healthcare system, the evaluation of the services provided to, and the outcomes of patients residing in the covered area. Particularly, the evaluation of health status of the population is deemed as a priority in the R.L. 7600, 20/12/2017, pp75 


\section{Consent for publication}

Not applicable.

\section{Availability of data and materials}

The datasets are not available as they belong to the Agency for Health Protection of the Province of Milan.

\section{Competing interests}

We declare no conflict of interest.

\section{Funding}

This work was supported by the Italian League Against Cancer. The funding body did not play any role in the design of the study and collection, analysis, and interpretation of data and in writing of the manuscript as it was an unrestricted funding.

\section{Authors' contributions}

AGM analysed, interpreted data and was a major contributor in the study design and in writing the manuscript. AO had a role in interpreting the data, writing and proofreading the final manuscript. CS had a role in interpreting the data, writing and proofreading the final manuscript. AGR supervised the project and was a major contributor in the study design, interpreting data and proofreading the manuscript. All authors have read and approved the manuscript.

\section{Acknowledgements:}

We would also like to sincerely thank and acknowledge those that have made significant contributions to making this effort happen: Luigi Bisanti, Patrizia Di Benedetto, Irene Accardi, Valentina Bruno, Schantal Alouche, the Cancer Registry personnel of the Epidemiology Unit and Anna Rita Silvestri, Enrica Tidone and the personnel of the Screening Unit of the Agency for Health Protection. The authors are indebted to the hospital staff who participated in the study ensuring collecting questionnaires.

\section{References}

1 OECD/EU; Paris: 2016. Health at a Glance: Europe 2016: State of Health in the EU Cycle.

2 Jacob L, Freyn M, Kalder M, Dinas K, Kostev K. Impact of tobacco smoking on the risk of developing 25 different cancers in the UK: a retrospective study of 422,010 patients followed for up to 30 years. Oncotarget. 2018 Apr 3;9(25):17420-17429. doi: 10.18632/oncotarget.24724. eCollection 2018 Apr 3. PubMed PMID: 29707117; PubMed Central PMCID: PMC5915125.

3 DOLL R, HILL AB. Smoking and carcinoma of the lung; preliminary report. Br Med J. 1950 Sep 30;2(4682):739-48. PubMed PMID: 14772469; PubMed Central PMCID: PMC2038856.

4 https://monographs.iarc.fr/wp-content/uploads/2018/06/mono100E-6.pdf

5 https://www.who.int/news-room/fact-sheets/detail/tobacco

6 Rodgman A, Perfetti TA (2009). Alphabetical Component Index. In: The Chemical Components of Tobacco and Tobacco Smoke. Rodgman A, Perfetti TA, editors. Boca Raton, FL: CRC Press, pp. 1483-1784.

7 IARC (2004a). Tobacco smoke and involuntary smoking. IARC Monogr Eval Carcinog Risks Hum, 83: 1-1438. PMID:15285078

8 http://apps.who.int/gho/data/node.sdg.3-a-viz?lang=en

9 WHO report on the global tobacco epidemic, 2019. Available at:

Page $12 / 14$ 
https://apps.who.int/iris/bitstream/handle/10665/326043/9789241516204-eng.pdf?ua=1

10 https://www.istat.it/it/files/2016/12/Asi-2016.pdf

11 de Groot PM, Wu CC, Carter BW, Munden RF. The epidemiology of lung cancer. Transl Lung Cancer Res. 2018;7(3):220-233. doi:10.21037/tlcr.2018.05.06

12 Rowland C, Eiser C, Rowe R, Danson S. The effect of smoking on health-related quality of life in lung cancer patients: a systematic review. BMJ Support Palliat Care. 2012 Dec;2(4):312-8. doi: 10.1136/bmjspcare-2011-000186. Epub 2012 Oct 12. Review. PubMed PMID: 24654214

13 Shikata S, Takemura Y. Secondhand smoke exposure and risk of lung cancer in Japan: a systematic review and meta-analysis of epidemiologic studies. Jpn J Clin Oncol. 2017 Mar 1;47(3):282. doi: 10.1093/jjco/hyw173. PubMed PMID: 27908979.

14 Altshuler B. Quantitative models for lung cancer induced by cigarette smoke. Environ Health Perspect. 1989 May;81:107-8; discussion 121-2. Review. PubMed PMID: 2667972; PubMed Central PMCID: PMC1567528.

15 Gaffney M, Altshuler B. Examination of the role of cigarette smoke in lung carcinogenesis using multistage models. J Natl Cancer Inst. 1988 Aug 17;80(12):925-31. PubMed PMID: 3398067.

16 Min KJ, Lee JK, So KA, Kim MK. Association Between Passive Smoking and the Risk of Cervical Intraepithelial Neoplasia 1 in Korean Women. J Epidemiol. 2018 Jan 5;28(1):48-53. doi: 10.2188/jea.JE20160118. Epub 2017 Oct 25. PubMed PMID: 29093300; PubMed Central PMCID: PMC5742379.

17 Trimble CL, Genkinger JM, Burke AE, Hoffman SC, Helzlsouer KJ, Diener-West M, Comstock GW, Alberg AJ. Active and passive cigarette smoking and the risk of cervical neoplasia. Obstet Gynecol. 2005 Jan;105(1):174-81. PubMed PMID: 15625160; PubMed Central PMCID: PMC3064987.

18 Fang JH, Yu XM, Zhang SH, Yang Y. Effect of smoking on high-grade cervical cancer in women on the basis of human papillomavirus infection studies. J Cancer Res Ther. 2018;14(Supplement):S184-S189. doi: 10.4103/0973-1482.179190. PubMed PMID: 29578171.

19 Samanic C, Kogevinas M, Dosemeci M, Malats N, Real FX, Garcia-Closas M, Serra C, Carrato A, García-Closas R, Sala M, Lloreta J, Tardón A, Rothman N, Silverman DT. Smoking and bladder cancer in Spain: effects of tobacco type, timing, environmental tobacco smoke, and gender. Cancer Epidemiol Biomarkers Prev. 2006 Jul;15(7):1348-54. Erratum in: Cancer Epidemiol Biomarkers Prev. 2006 Aug;15(8):1568. PubMed PMID: 16835335

20 Baris D, Karagas MR, Verrill C, Johnson A, Andrew AS, Marsit CJ, Schwenn M, Colt JS, Cherala S, Samanic C, Waddell R, Cantor KP, Schned A, Rothman N, Lubin J, Fraumeni JF Jr, Hoover RN, Kelsey KT, Silverman DT. A case-control study of smoking and bladder cancer risk: emergent patterns over time. J Natl Cancer Inst. 2009 Nov; 101(22):1553-61. doi: 10.1093/jnci/djp361. Epub 2009 Nov 16. PubMed PMID: 19917915; PubMed Central PMCID: PMC2778671.

21 Ding Y, Yu C, Han Z, Xu S, Li D, Meng X, Chen D. Environmental tobacco smoke and pancreatic cancer: a case-control study. Int J Clin Exp Med. 2015 Sep 15;8(9):16729-32. eCollection 2015. PubMed PMID: 26629212; PubMed Central PMCID: PMC4659100.

22 Villeneuve PJ, Johnson KC, Mao Y, Hanley AJ; Canadian Cancer Registries Research Group.. Environmental tobacco smoke and the risk of pancreatic cancer: findings from a Canadian population-based case-control study. Can J Public Health. 2004 Jan-Feb;95(1):32-7. PubMed PMID: 14768739.

23 Bao Y, Giovannucci E, Fuchs CS, Michaud DS. Passive smoking and pancreatic cancer in women: a prospective cohort study.Cancer Epidemiol Biomarkers Prev. 2009 Aug;18(8):2292-6. doi: 10.1158/1055-9965.EPI-09-0352. Epub 2009 Jul 14. PubMed PMID: 19602702; PubMed Central PMCID: PMC2927115.

24 Simarak S, de Jong UW, Breslow N, Dahl CJ, Ruckphaopunt K, Scheelings P, Maclennan R. Cancer of the oral cavity, pharynx/larynx and lung in North Thailand: case-control study and analysis of cigar smoke. Br J Cancer. 1977 Jul;36(1):130-40. PubMed PMID: 19028; PubMed Central PMCID: PMC2025440.

25 Hashibe M, Brennan P, Benhamou S, Castellsague X, Chen C, Curado MP, Dal Maso L, Daudt AW, Fabianova E, Fernandez L, Wünsch-Filho V, Franceschi S, Hayes RB, Herrero R, Koifman S, La Vecchia C, Lazarus P, Levi F, Mates D, Matos E, Menezes A, Muscat J, Eluf-Neto J, Olshan AF, Rudnai P, Schwartz SM, Smith E, Sturgis EM, Szeszenia-Dabrowska N, Talamini R, Wei Q, Winn DM, Zaridze D, Zatonski W, Zhang ZF, Berthiller J, Boffetta P. Alcohol drinking in never users of tobacco, cigarette smoking in never drinkers, and the risk of head and neck cancer: pooled analysis in the International Head and Neck Cancer Epidemiology Consortium. J Natl Cancer Inst. 2007 May 16;99(10):777-89. Erratum in: J Natl Cancer Inst. 2008 Feb 6;100(3):225. Fernandez, Leticia [added]. PubMed PMID: 17505073. 
26 Bravi F, Decarli A, Russo AG. Risk factors for breast cancer in a cohort of mammographic screening program: a nested case-control study within the FRiCaM study. Cancer Med. 2018 May;7(5):2145-2152. doi: 10.1002/cam4.1427. Epub 2018 Apr 14. PubMed PMID: 29654663; PubMed Central PMCID: PMC5943434.

27 Fernandes TP, Silverstein SM, Almeida NL, Santos NA. Visual impairments in tobacco use disorder. Psychiatry Res. 2019 Jan;271:60-67. doi: 10.1016/j.psychres.2018.11.024. Epub 2018 Nov 14. PubMed PMID: 30469090.

28 Giovannucci E, Rimm EB, Stampfer MJ, Colditz GA, Ascherio A, Kearney J, Willett WC. A prospective study of cigarette smoking and risk of colorectal adenoma and colorectal cancer in U.S. men. J Natl Cancer Inst. 1994 Feb 2;86(3):183-91. doi: 10.1093/jnci/86.3.183. PubMed PMID: 8283490 .

29 Giovannucci E, Colditz GA, Stampfer MJ, Hunter D, Rosner BA, Willett WC, Speizer FE. A prospective study of cigarette smoking and risk of colorectal adenoma and colorectal cancer in U.S. women. J Natl Cancer Inst. 1994 Feb 2;86(3):192-9. doi: 10.1093/jnci/86.3.192. PubMed PMID: 8283491.

30 Chao A, Thun MJ, Jacobs EJ, Henley SJ, Rodriguez C, Calle EE. Cigarette smoking and colorectal cancer mortality in the cancer prevention study II. J Natl Cancer Inst. 2000 Dec 6;92(23):1888-96. doi: 10.1093/jnci/92.23.1888. PubMed PMID: 11106680.

31 Knekt P, Hakama M, Järvinen R, Pukkala E, Heliövaara M. Smoking and risk of colorectal cancer. Br J Cancer. 1998 Jul;78(1):136-9. doi: 10.1038/bjc.1998.455. PubMed PMID: 9662264; PubMed Central PMCID: PMC2062953

32 Peppone LJ, Reid ME, Moysich KB, Morrow GR, Jean-Pierre P, Mohile SG, Darling TV, Hyland A. The effect of secondhand smoke exposure on the association between active cigarette smoking and colorectal cancer. Cancer Causes Control. 2010 Aug;21(8):1247-55. doi: 10.1007/s10552010-9552-3. Epub 2010 Apr 8. PubMed PMID: 20376547; PubMed Central PMCID: PMC3085188.

33 Slattery ML, West DW, Robison LM, French TK, Ford MH, Schuman KL, Sorenson AW. Tobacco, alcohol, coffee, and caffeine as risk factors for colon cancer in a low-risk population. Epidemiology. 1990 Mar;1(2):141-5. PubMed PMID: 2073501.

34 Yang C, Wang X, Huang CH, Yuan WJ, Chen ZH. Passive Smoking and Risk of Colorectal Cancer: A Meta-analysis of Observational Studies. Asia Pac J Public Health. 2016 Jul;28(5):394-403. doi: 10.1177/1010539516650724. Epub 2016 May 23. Review. PubMed PMID: 27217428.

35 Le Marchand L, Wilkens LR, Kolonel LN, Hankin JH, Lyu LC. Associations of sedentary lifestyle, obesity, smoking, alcohol use, and diabetes with the risk of colorectal cancer. Cancer Res. 1997 Nov 1;57(21):4787-94. PubMed PMID: 9354440.

36 Yang HP, Brinton LA, Platz EA, Lissowska J, Lacey JV Jr, Sherman ME, Peplonska B, Garcia-Closas M. Active and passive cigarette smoking and the risk of endometrial cancer in Poland. Eur J Cancer. 2010 Mar;46(4):690-6. doi: 10.1016/j.ejca.2009.11.015. Epub 2009 Dec 28. PubMed PMID: 20036529; PubMed Central PMCID: PMC2851155.

37 Faber MT, Kjær SK, Dehlendorff C, Chang-Claude J, Andersen KK, Høgdall E, Webb PM, Jordan SJ, Rossing MA, Doherty JA, Lurie G, Thompson PJ, Carney ME, Goodman MT, Ness RB, Modugno F, Edwards RP, Bunker CH, Goode EL, Fridley BL, Vierkant RA, Larson MC, Schildkraut J, Cramer DW, Terry KL, Vitonis AF, Bandera EV, Olson SH, King M, Chandran U, Kiemeney LA, Massuger LF, van Altena AM, Vermeulen SH, Brinton L, Wentzensen N, Lissowska J, Yang HP, Moysich KB, Odunsi K, Kasza K, Odunsi-Akanji O, Song H, Pharaoh P, Shah M, Whittemore AS, McGuire V, Sieh W, Sutphen R, Menon U, Gayther SA, Ramus SJ, Gentry-Maharaj A, Pearce CL, Wu AH, Pike MC, Risch HA, Jensen A. Cigarette smoking and risk of ovarian cancer: a pooled analysis of 21 case-control studies. Cancer Causes Control. 2013 May;24(5):989-1004. doi: 10.1007/s10552-0130174-4. Epub 2013 Mar 2. PubMed PMID: 23456270; PubMed Central PMCID: PMC3818570.

38 Beral V, Gaitskell K, Hermon C, Moser K, Reeves G, Peto R. Ovarian cancer and smoking: individual participant meta-analysis including 28,114 women with ovarian cancer from 51 epidemiological studies. Lancet Oncol. 2012 Sep;13(9):946-56. doi: 10.1016/S1470-2045(12)70322-4. Epub 2012 Aug 3. Review. PubMed PMID: 22863523; PubMed Central PMCID: PMC3431503. 